\title{
Analysis of Ionic Domains on a Proton Exchange Membrane using a Numerical Approximation Model based on Electrostatic Force Microscopy
}

\author{
Byungrak Son ${ }^{1}$, JaeHyoung Park ${ }^{2}$ and Osung Kwon ${ }^{3, *}$ \\ 1 Division of Energy Technology, DGIST, Daegu 42988, Korea; brson@dgist.ac.kr (B.S.) \\ 2 Corporate Research Center, HygenPower Co.,Ltd., Daegu 42988, Korea; chris@hygenpower.com (J.P.) \\ 3 Tabula Rasa College, Keimyung University, Daegu 42601, Korea; 11502@gw.kmu.ac.kr (O.K.) \\ * Correspondence: 11502@gw.kmu.ac.kr; Tel.: +82-53-580-5657
}

\begin{abstract}
Understanding the ionic channel network of proton exchange membranes, which dictate fuel cell performance, is crucial when developing proton exchange membrane fuel cells. However, itis difficult to characterize due to complicated nano structure and differing changes to their structure with different amounts of water uptake. Electrostatic force microscopy (EFM) can map surface charge distribution as nano special resolution by measuring the electrostatic force between a vibrating conductive tip and a charged surface under an applied voltage, . In this study, the ionic channel network of a proton exchange membrane is analyzed using EFM. A mathematical approximation model of the ionic channel network is first derived, to explain changes in force gradient on the surface using EFM. The phase lag of dry and wet Nafion under stepwise changes to bias voltage is then measured. Based on the model, variations in the ionic channel network of Nafion with different amounts of water uptake are analyzed numerically. The mean surface charge density of both membranes, which is connected with the ionic channel network, is calculated using the model. The results show that the difference between the mean surface charge of the dry and wet membranes is consistent with the variation in their proton conductivity.
\end{abstract}

Keywords: electrostatic force microscopy; proton exhange membrane; ionic domain; surface charge density; PEMFC

\section{Introduction}

Proton exchange membrane fuel cells (PEMFCs) are a core technology of green energy devices for several reasons; they do not emit carbon dioxide, can operate continuously in different environmental conditions without changes to performance, and they have a relatively high energy conversion efficiency. However, a number of hurdles must be overcome before their adoption is feasible, such as their high cost, low reliability, and a lack of hydrogen gas. The need to address the low reliability is particularly urgent. However, this problem has proved very difficult to solve. The reason for this difficulty is that a proton exchange membrane's reliability is connected to its morphological structure.

Proton exchange membranes typically work as proton conductors because of their heterogeneous structures, which is the combination of a hydrophobic backbone with hydrophilic sulfonic acid groups. The relatively poor reliability of proton exchange membranes is caused directly by their poor mechanical and thermal properties [1-3] Although hydration is crucial for enhancing the proton conductivity of Nafion, it is the cause of the material's weak mechanical properties [2]. Novel membranes and Nafion- 
based composites have been developed to address the problem of poor thermal and mechanical properties [4-6].

Understanding the morphological structure of Nafion is as important as developing novel membranes, because the ability of proton movement to mirror morphological structures such as the ionic channel network is the essential function of the proton exchange membrane. Since the 1980s, many research groups have studied to understand the morphological structure of Nafion [7-9]. Gierke et al. introduced a cluster-network model of Nafionbased on small-angle X-ray scattering and wide-angle X-ray scattering measurements [10]. According to this model, the ionic channel network is formed by the hydration of ionic clusters, which, in dry conditions, consist of sulfonic acid groups in a semicrystalline matrix. These ionic clusters are spheres with a $4 \mathrm{~nm}$ diameter in an inverted micellar structure, with a narrow $1 \mathrm{~nm}$ channel connecting each cluster. The ionic channel network becomes more widely interconnected as water uptake in the Nafion increases, and the structure becomes more complex as protons move through the network.

Following Gierke's study, core-shell model [11], rod-like model [12], and cylindrical water channel [13] $]$ were proposed. The most recent of these is Klaus and Chen's cylindrical water channel model [13], based on simulation studies conducted using existing scattering data. According to Klaus and Chen, cylindrical crystallites of 2-5 nm, and cylindrical water channels with a radius of $2-3 \mathrm{~nm}$ are formed in the polymer matrix. Each cylindrical water channel increases in size as the volume of water contained in Nafion increases, and the existence of cylindrical crystallites contributes to the mechanical strength of Nafion.

Despite numerous studies on the morphology of Nafion, the structure of the ionic channel network is still unclear. Thus, the morphology of Nafion has been studied using atomic force microscopy (AFM), a technique that can map topography at nanoscale resolutions [14-16]. AFM can also be used for studying a material's electrical, magnetic, and mechanical properties. With this technique, surface topography is mapped to a nanoscale resolution using a scanning tip that can move in the vertical direction. Physical properties are mapped using a vibrating tip technique. Here, the tip is oscillated sinusoidally as the surface is scanned, and variations in the amplitude and frequency of vibration are mapped $[\underline{17}, \underline{18}]$. Changes in the phase of vibration relates by local mechanical properties of the surface, such as elasticity and viscoelasticity $[\underline{19}, \underline{20}]$. The electric properties of a surface can also be characterized using electrostatic force microscopy (EFM), or Kelvin probe microscopy [21].

EFM has great potential to understand surface electrical characteristic. Thus, it is widely used in studies of the surface charge distribution of locally charged materials [22, 23]. Mélin et al. developed an analytical model for estimating the amount of charge stored on a surface using EFM [르] . Here, they assumed that the tip and sample surface created a parallel-plate capacitor, and determined the amount of stored charge by calculating the capacitive force. They subsequently expanded this model to consider the tip and sample surface as other capacitor shapes. Han et al. studied the movement and diffusion of natural and injected charge using EFM, to understand the interface of a nano-dielectric [19]. They analyzed EFM images using a widely-accepted methodical model [르], to explain local charge movement at the SiO2/LDPE boundary. Shen et al. characterized the degree of reduction of uniformly monolayered reduced graphene oxide sheets at the nanoscale, by analyzing EFM phase signals produced by scanning the graphene oxide sheets with different bias voltages applied to the tip. The map can be interpreted mathematically [25] .

Numerical approaches for understanding the morphology of proton exchange membranes, though the local charge density and dielectric constant are based on AFM. Numerous current-sensing AFM and EFM studies have been conducted, with many meaningful results [ㄹ-29]. 
In this study, EFM is used to analyze the movement of free charges, to elucidate ionic channel morphology. For this, we derived a numerical model of a proton exchange membrane based on Zhang et al's study [26]. Using this numerical model, we characterize the ionic channel network of proton exchange membranes with different amounts of water uptake. We also extract quantitative information relating the ionic channel network to the proton exchange membrane. Furthermore, we attempt to provide a general model for interpreting changes in the morphology of a proton exchange membrane.

\section{Experimental Setup and Model Development}

Nafion 212 membranes were studied in two different conditions in our experiments. These conditions were prepared as follows. The first membrane, called the dry membrane, was inserted an oven, with the temperature maintained at $80{ }^{\circ} \mathrm{C}$ overnight, while the second membrane, called the wet membrane, was soaked in water overnight. Before measurement, the dry membrane was exposed in ambient conditions for $2 \mathrm{~h}$, while the wet membrane was soaked in water.

Both membranes were mapped and analyzed systematically in several steps. First, each membrane was scanned at a frequency of $1 \mathrm{~Hz}$ as the sample bias voltage was changed from $-3 \mathrm{~V}$ to $3 \mathrm{~V}$ in $1 \mathrm{~V}$ intervals. Phase images and topography are mapped simultaneously in this step. The mean phase lag value of each image was subsequently calculated and plotted. Finally, these mean phase values were analyzed using an approximation model based on Zhang et al's study [26].

An electrical interaction occurs when a bias voltage is applied between the tip and the sample surface, as the dielectric sample becomes polarized. The capacitive force that is induced between the tip and the sample surface can be expressed as,

$$
\mathrm{F}=\frac{1}{2} \frac{\partial C}{\partial z} V^{2},
$$

where $\mathrm{F}$ is the capacitive force, $\mathrm{C}$ is the capacitance of the space between the tip and the sample, $V$ is the applied voltage, and $z$ is the distance between the tip and the sample surface. The capacitance of the tip $\left(C_{\text {tip }}\right)$, which is modelled as a plate, is

$$
C_{\text {Tip }} \cong \pi \varepsilon_{0} R_{\text {tip }}^{2},
$$

where $R_{\text {tip }}$ is the radius of the tip, and $\varepsilon_{0}$ is the permittivity of free space. From the capacitance equation, the charge accumulated in the tip is

$$
Q_{\text {Tip }}=C_{\text {Tip }} V .
$$

The tip and sample create a nano sized parallel-plate capacitor filled with air and Nafion, as shown in Figure 1. 


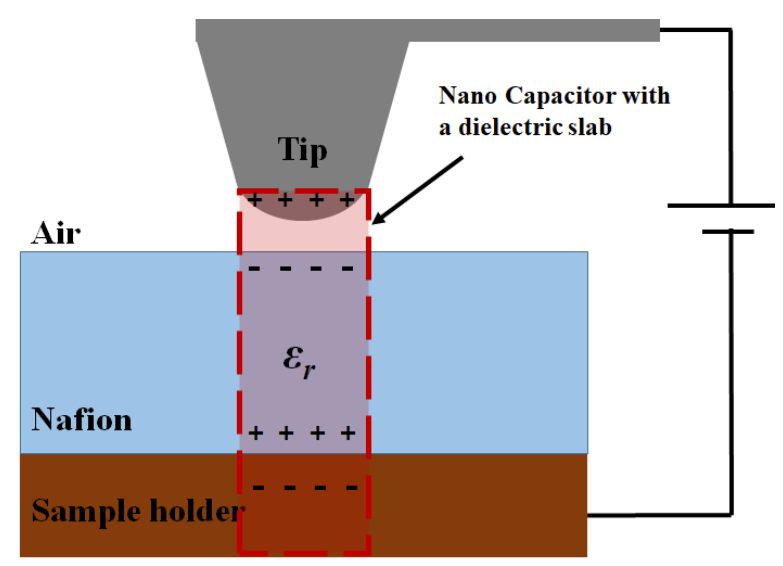

Figure 1. Configuration of the conductive tip and Nafion attached to the sample holder, explaining the origin of the capacitor model.

The capacitance of this parallel-plate capacitor is calculated as,

$$
\mathrm{C}=\frac{Q_{\text {tip }}}{V}
$$

where,

$$
V=\frac{Q_{t i p}}{\varepsilon_{0} S}\left(z+\frac{t}{\varepsilon_{r}}\right),
$$

$S$ is the area under the tip, $t$ is the thickness of the membrane, and $\varepsilon_{r}$ is its relative permittivity. Then,

$$
\mathrm{C}=\frac{\varepsilon_{0} A}{\left(z+\frac{t}{\varepsilon_{r}}\right)},
$$

where $A$ is the area of the tip,

$$
\frac{\partial \mathrm{C}}{\partial \mathrm{z}}=-\frac{2 \varepsilon_{0} S}{\left(z+\frac{t}{\varepsilon_{r}}\right)^{2}}
$$

and the capacitance force is,

$$
\mathrm{F}_{C}=-\frac{1}{2} \frac{2 \varepsilon_{0} S}{\left(z+\frac{t}{\varepsilon_{r}}\right)^{2}} V^{2}
$$

Local free charges exist in Nafion due to the ionic domain. Thus, an electrostatic force is also induced between the tip and the sample surface, given as,

$$
F_{f}=\frac{1}{4 \pi \varepsilon_{0}} \frac{Q_{\text {free }} Q_{\text {tip }}}{z^{2}}=\frac{Q_{\text {free }}}{4 z^{2}} R_{\text {tip }}^{2} V,
$$

where (2) has been used to reduce the number of charge variables. Hence, the net force between the tip and the sample surface is the sum of the capacitance force and the electrostatic force, given as,

$$
\mathrm{F}=-\frac{1}{2} \frac{2 \varepsilon_{0} S}{\left(z+\frac{t}{\varepsilon_{r}}\right)^{2}} V^{2}+\frac{Q_{\text {free }}}{4 z^{2}} R_{\text {tip }}^{2} V,
$$

and the force gradient is, 


$$
\frac{\partial \mathrm{F}}{\partial \mathrm{z}}=\frac{\pi \varepsilon_{0} R_{\text {tip }}^{2}}{\left(z+\frac{t}{\varepsilon_{r}}\right)^{3}} V^{2}-\frac{Q_{\text {free }}}{2 z^{3}} R_{\text {tip }}^{2} V .
$$

The frequency shift of Nafion is expressed as,

$$
\Delta \mathrm{f} \cong-\frac{F^{\prime}}{2 k} f_{0}=-\frac{\pi \varepsilon_{0} R_{\text {tip }}^{2}}{2 k\left(z+\frac{t}{\varepsilon_{r}}\right)^{3}} f_{0} V^{2}+\frac{Q_{\text {free }}}{4 k z^{3}} R_{\text {tip }}^{2} f_{0} V,
$$

while phase shift is given as,

$$
\Delta \emptyset \cong \mathrm{A} \Delta \mathrm{f}=-\frac{\pi \varepsilon_{0} A R_{t i p}^{2} f_{0}}{2 k\left(z+\frac{t}{\varepsilon_{r}}\right)^{3}} V^{2}+\frac{Q_{f r e e} A R_{t i p}^{2} f_{0}}{4 k z^{3}} V
$$

Simples $\mathrm{k}$ and $\mathrm{f}_{0}$ represent spring constant of a tip and resonance frequency.

If a sample is uniform and does not contain local surface charges, the polarity of the surface charge of the dielectric sample is opposite to that of the tip charge. Hence, if a positive bias voltage is applied, the tip charge is negative and the sample surface is positively charged, and vice versa. Thus, the force between the tip and the sample surface is always attractive, as shown in Figure 2, even if the polarity of the bias voltage is changed. In this case, the first term in (13) is dominant. Thus, there is a parabolic relationship between the phase shift and bias voltage, as shown in Figure 2, which also depends on $\varepsilon r$. In identical experimental conditions, if the sample is homogeneous, the phase shift is similar in all scanned areas because the relative permittivity is the same. However, the phase shift changes in heterogeneous material due to local differences in relative permittivity. When experimental conditions such as temperature and humidity change, different phase shifts are measured due to these local changes in relative permittivity.
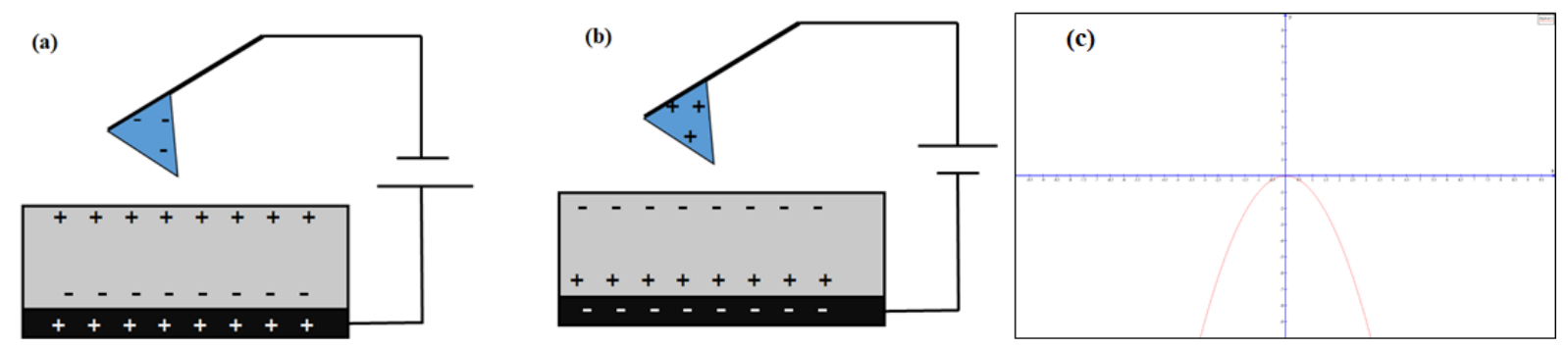

Figure 2. Charge distribution in a system with a (a) positive, and (b) negative bias voltage applied. (c) Variation of phase lag value with bias voltage.

The behavior of ion exchange membranes can be explained by the combination of the PTFE backbone and the ionic channel network created by the interconnection of ionic clusters, which consist of sulfonic acid groups. When water binds with the negativelycharged sulfonic acid groups, protons are solvated, and free charges exist in the membrane. Since locally charged regions exist in ion exchange membranes, the phase shift is affected by both the first and second terms of (13). Here, Q Qree is the local charge related to the ionic cluster, in this case, proton movement into the ionic channel network. The distribution of ionic channel networks on a surface is random, and changes with surface hydration. Thus, characterization of ionic clusters in an ion exchange membrane is complicated, and it is even more difficult in composite membranes. However, measuring force gradient, which is related to free charge, provides a simple quantitative method for 
characterizing the ionic channel network. Quantitative information on the homogeneity and distribution of the ionic domains on a membrane can be provided by estimating local variations in free charge and relative permittivity.

\section{Results}

Figure 3 depicts EFM phase images of dry and wet Nafion with bias voltage swept from $-3 \mathrm{~V}$ to $3 \mathrm{~V}$ in $1 \mathrm{~V}$ steps. The colors in the image indicate the phase lag value, which represents the force gradient. From the image, the color is darker with bias voltage. When the same bias voltage is maintained, color is uniform except in the left side of the image. This indicates that the areas that have homogeneous morphological characteristics have similar phase lag values. However, the phase lag in each colored region does not follow the parabolic shape that is typical of changes to force gradient due to induced charge, as was shown in Fig. 2.

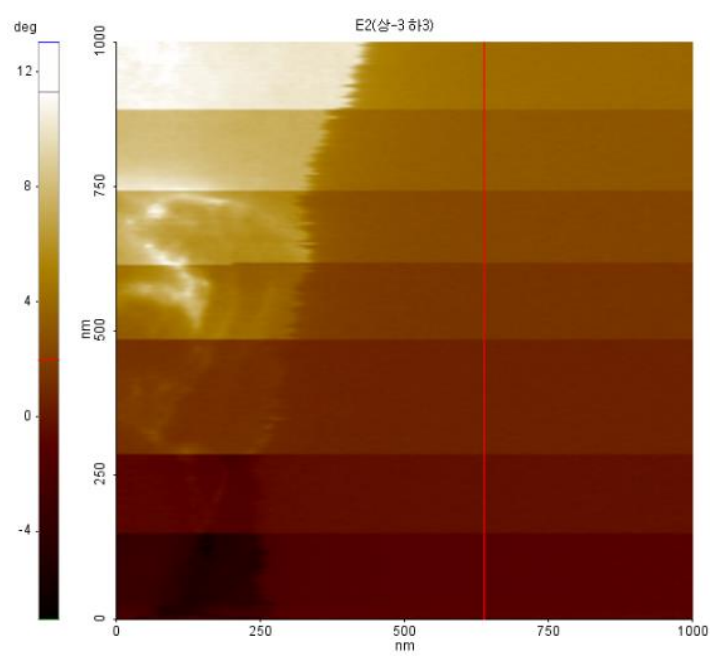

(a)

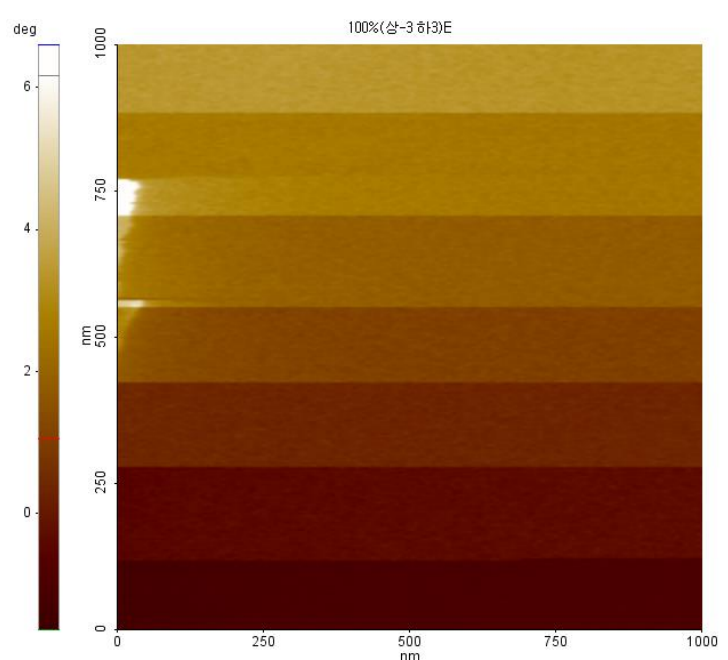

(b)

Figure 3. EFM image of (a) dry and (b) wet membrane.

Figure 4 depicts the line profiles of the dry and wet proton exchange membranes, providing numerical information on the phase shift at each bias voltage. Both images show small changes in phase shift of $\sim 0.2^{\circ}$ when the same bios voltage is maintained, and relatively large phase shifts of $1^{\circ}$ when bias voltage changes. Both membranes have positive phase shift values between $-3 \mathrm{~V}$ and $0 \mathrm{~V}$, indicating that the net electrostatic force between the tip and the sample surface is repulsive. In the negative bias voltage configuration, the tip is positively charged, and, typically, the force between the tip and the sample surface is attractive, due to the negatively-polarized membrane surface. The result depicts the opposite phenomenon, implying that the sample surface is positively charged. For negative bias voltages, phase lag values are slightly higher for dry membranes than they are for wet membranes. Phase shift is negative between $2 \mathrm{~V}$ and 3 $\mathrm{V}$, indicating that the force is in the attractive regime. With these bias voltages, both membranes have similar phase lag values. 


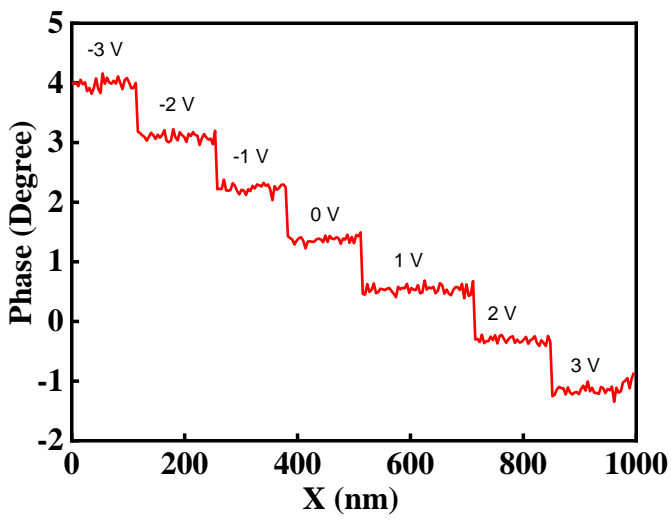

(a)

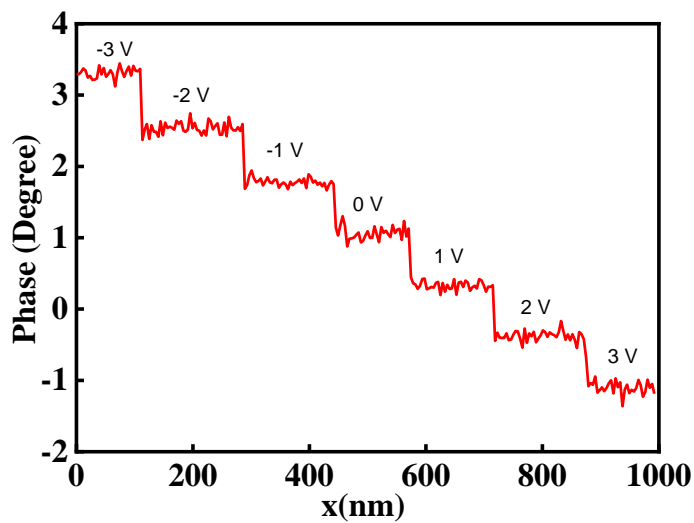

(b)

Figure 4. Line profile of (a) dry, and (b) wet membrane

For more detailed analysis, the mean phase value at each bios voltage was plotted for both the dry and wet membranes. From this, it can be observed that the phase lag value varies linearly with bias voltage with both membranes, as shown in Figure 5 . There are locally charged regions on the membrane, the behavior of which is characterized by the second term in (13). As phase lag is the sum of both terms in (13), a positive phase lag value indicates that the second term, relating to local surface charge, is dominant. When bias voltage is reduced, the phase lag decreases; with the wet membrane, phase lag values of $3.4^{\circ}, 2.5^{\circ}$, and $1.8^{\circ}$ were noted at bias voltages of $-3 \mathrm{~V},-2 \mathrm{~V}$, and $-1 \mathrm{~V}$, indicating that both terms in the equation decreased as bias voltage was reduced. With the dry membrane, the phase lag values were $4.0^{\circ}, 3.4^{\circ}$, and $2.5^{\circ}$ at $-3 \mathrm{~V},-2 \mathrm{~V}$, and $-1 \mathrm{~V}$, respectively. Wet membranes typically have higher proton conductivities than dry membranes, and a high ionic channel network density, due to their creation of an ionic channel network. The difference between the phase lag values of wet and dry membranes is thus connected to the second term in (13). At $1 \mathrm{~V}$, this value is close to zero. In contrast, at $2 \mathrm{~V}$ and $3 \mathrm{~V}$, both membranes have similar negative phase values. Both membranes has similar phase values at $2 \mathrm{~V}$ and $3 \mathrm{~V}$. Specifically, dry and wet membranes have the same the lag value at $3 \mathrm{~V}$. This result implies that the electrical interaction is only between the charged tip and the polarized surface charge. Thus, the second term in (13) does not have any effect on the phase lag in this case. 


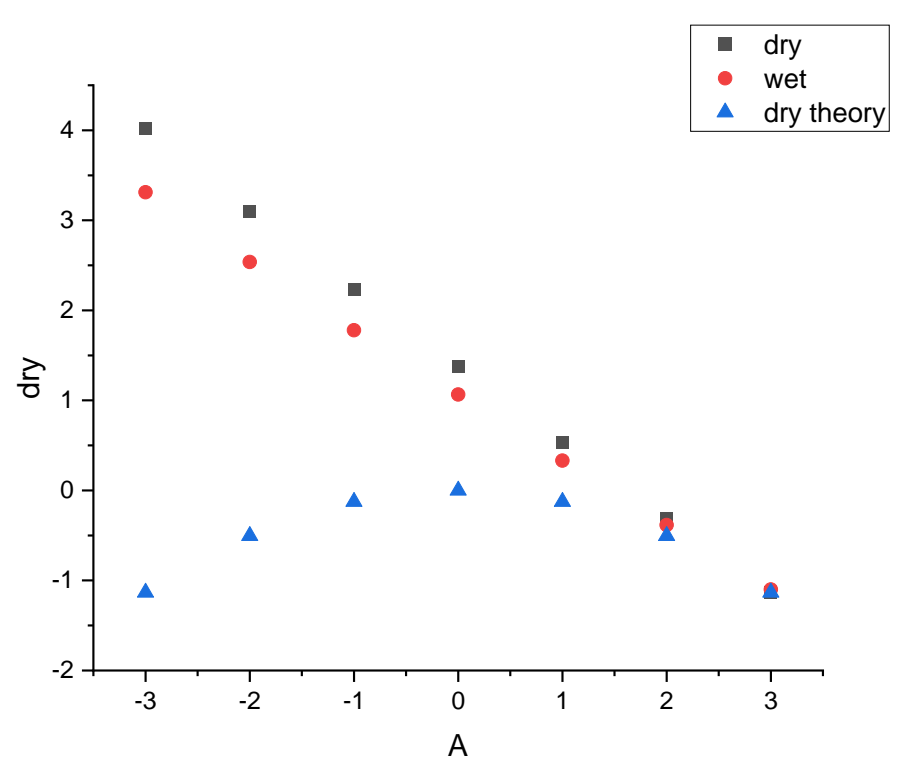

Figure 5. Variation of phase lag with bias voltage for different membrane conditions

\section{Analysis and Conclusion}

Local charge density, which reflects the ionic channel network, can be approximated based the first term and second term of (13). To do this, the phase lag value at each bias voltage must be related to a microscopic electrostatic phenomenon. To understand the generation of positive phase lag at negative sample bias voltage, the operation of a tip when bias voltage is applied during scanning must be analyzed. There is typically a water layer between the tip and the sample surface. When the bias voltage is applied, hydrolysis occurs, hydrogen is produced, and protons are created because of the Pt-coated tip. Figure 6 depicts the local variation in the current flowing through the $\mathrm{Pt}$ tip and the half membrane electrode assembly as bias voltage is swept. Current flows when the magnitude of the bias voltage is larger than $1.5 \mathrm{~V}$, indicating that protons are created when a voltage is applied to the Pt tip. 


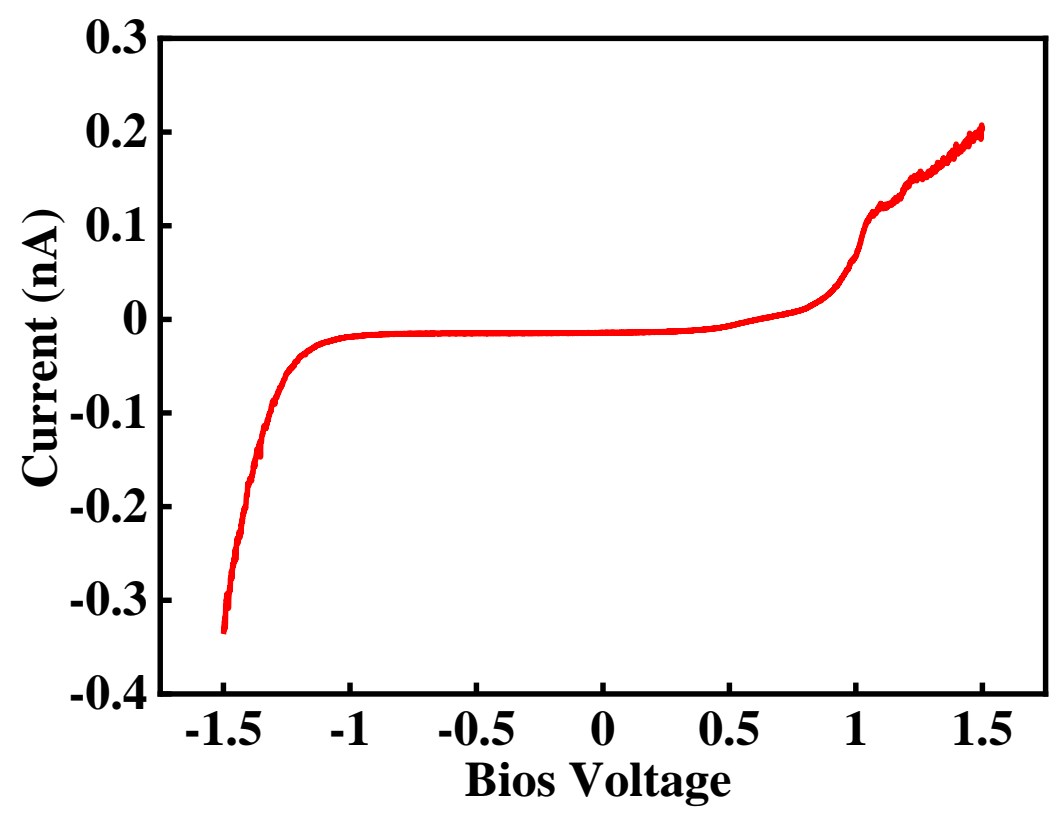

Figure 6. Variation of current with bias voltage applied to the Pt tip

The phase lag generated at negative bias voltages includes a contribution from the interaction between released protons and the ionic domains on the membrane surface. As the membrane is negatively charged, due to polarization, it attracts protons that cover its surface. Thus, positive phase lag values are measured, because a repulsive force is induced between the positively-charged tip and the proton-covered surface. The magnitude of the repulsive force is related to the density of the activated ionic channel network. When water uptake in the membrane increases, an ionic channel network is developed, as the number of interconnections between the ionic channels grows. Protons are accelerated into the ionic channel by the external electric field, as shown in Figure 7. The number of ionic domains increases as the number of protons on the membrane surface decreases. Thus, there is a reciprocal relationship between the repulsive force between the tip and the membrane and the area of the ionic domain.
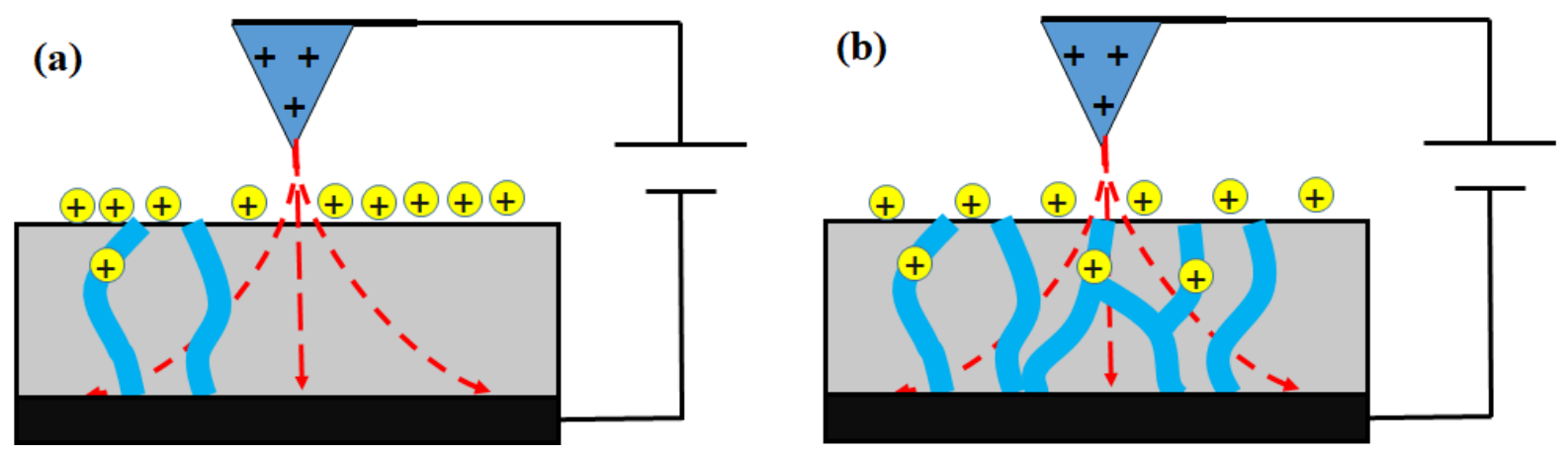

Figure 7. Proton movement into the ionic channel with (a) negative, and (b) positive sample bias voltages

Table 1 summarizes the mean phase lag values for dry and wet membranes, and the values when there are no protons on the membrane surface. The latter value is calculated 
using only negative bias voltages. With both dry and wet membranes, the phase lag increased with the bias voltage, which can be explained as proton generation increasing due to hydrolysis. At all negative bias voltages, dry membranes have a larger phase lag value than wet membranes, which is consistent with our assumptions. Hence, the area of the ionic domain on the membrane can be approximated using phase lag difference.

The net electrical charge of the protons at each bias voltage and membrane condition was estimated using (13). This approximation is conducted in several steps. First, because the phase lag value obtained for each membrane includes a contribution due to polarization-induced charge, the phase lag when there are no protons on the membrane surface is subtracted from this value. Then, a tip radius is calculated for each membrane using the blind tip reconstruction method [30]. Finally, the net charge of the protons is calculated using the second term of (13).

Table 1. Mean phase lag value of each membrane

\begin{tabular}{|r|r|r|r|r|r|}
\hline $\begin{array}{l}\text { Bios } \\
\text { voltage } \\
\text { (V) }\end{array}$ & $\begin{array}{l}\text { Dry } \\
\text { membrane } \\
\text { (Degree) }\end{array}$ & $\begin{array}{l}\text { Wet } \\
\text { membrane } \\
\text { (Degree) }\end{array}$ & $\begin{array}{l}\text { No proton } \\
\text { (Degree) }\end{array}$ & $\begin{array}{l}\text { Phase lag difference } \\
\text { between wet membrane } \\
\text { and no proton }\end{array}$ & \multicolumn{2}{l}{$\begin{array}{l}\text { Phase lag difference } \\
\text { between dry membrane } \\
\text { and no proton }\end{array}$} \\
\hline-3 & 4.02 & 3.31 & -1.134 & 5.15 & 4.45 \\
\hline-2 & 3.10 & 2.54 & -0.504 & 3.60 & 3.04 \\
\hline-1 & 2.24 & 1.78 & -0.126 & 2.36 & 1.90 \\
\hline
\end{tabular}

The results of calculation for net charge are summarized in Table 2 . In the dry membrane, the net charge is $8.71 \times 10^{-18} \mathrm{C}, 6.07 \times 10^{-18} \mathrm{C}$, and $3.99 \times 10^{-18} \mathrm{C}$ at $-3 \mathrm{~V},-2 \mathrm{~V}$, and $-1 \mathrm{~V}$, respectively. Hence, the net charge increases as bias voltage is increased. The value at $-1 \mathrm{~V}$ is much smaller than the net charge at the other voltages, due to the relatively small amount of proton generation at $-1 \mathrm{~V}$. This is consistent with the variation in local current with a swept bias voltage, as shown in Figure 6. However, the latter result does not provide absolute numerical information about the ionic domain. In the wet membrane, the net charge is $1.87 \times 10^{-18} \mathrm{C}, 1.28 \times 10^{-18} \mathrm{C}$, and $8.06 \times 10^{-18} \mathrm{C}$ at $-3 \mathrm{~V},-2 \mathrm{~V}$, and $-1 \mathrm{~V}$, respectively. These results show a similar trend to that observed with the dry membranes. However, the amount of electrical charge is much smaller than with dry membranes, possibly due to the partial movement of protons into the ionic channels. This result indicates that wet membranes have a larger ionic domain than dry membranes. Here, the repulsive force is due only to the protons that do not move into the ionic channel network. The difference between the net charge of dry and wet membranes is similar at each bias voltage, and is $\sim 79 \%-80 \%$. This result implies that $80 \%$ of the liberated protons move into the wet membrane, only $20 \%$ of protons interact with the tip, and this ratio is independent of bias voltage. From this, it can be surmised that the area of the ionic channels on the surface of a wet membrane increases by $\sim 80 \%$ compared with that on a dry membrane. Previous experimental results have shown that there is an approximately $80 \%$ difference between proton conductivity in ambient conditions and fully humid conditions [1ㅣ 32]. Hence, our calculations are consistent with the literature.

Table 2. Net charge of each membrane

\begin{tabular}{|r|r|r|r|}
\hline Bios voltage (V) & Net charge of dry membrane (C) & Net charge of wet membrane (C) & \% difference \\
\hline-3 & $8.71 \times 10^{-18}$ & $1.87 \times 10^{-18}$ & 78.5 \\
\hline-2 & $6.07 \times 10^{-18}$ & $1.28 \times 10^{-18}$ & 78.9 \\
\hline-1 & $3.99 \times 10^{-18}$ & $0.81 \times 10^{-18}$ & 79.8 \\
\hline
\end{tabular}


In this study, we determined the local charge density of a proton exchange membrane, which is directly related to the ionic domain, using an approximation model, and phase lag values obtained from electrostatic force microscopy. We also studied the variation of ionic channel networks in Nafion membranes in different conditions, with the results agreeing well with those of previous studies [31]. The enhancement of proton conductivity is prime purpose of developing the proton exchange membranes. Proton conductivity is governed by morphological structure of ionic channel network. Thus, the characterization of ionic channel network is mandatory of developing the novel proton exchange membranes. The numerical approximation model for local charge density derived using electrostatic force microscopy is become an important tool for characterize novel proton exchange membrane.

Author Contributions: Conceptualization, B.S. and O.K.; validation, B.S., J.P. and O.K.; investigation, B.S., J.P. and O.K.; writing - original draft preparation, O.K.; writing-review and editing, B.S.; supervision, O.K.; project administration, O.K.; All authors have read and agreed to the published version of the manuscript.

Funding: This work was supported by the DGIST R\&D Program of the Ministry of Science and ICT (21-ET-08) and Research Institute R\&DB Program through the Ministry of Science and ICT (2020-DG-RD0031-01-20).

Institutional Review Board Statement: Not applicable.

Informed Consent Statement: Not applicable.

Data Availability Statement: Data is contained within the article.

Conflicts of Interest: The authors declare no conflict of interest. The funders had no role in the design of the study; in the collection, analyses, or interpretation of data; in the writing of the manuscript, or in the decision to publish the results.

\section{References}

1. Kundu, S., et al., Mechanical properties of Nafion (TM) electrolyte membranes under hydrated conditions. Polymer, 2005. 46(25): p. 11707-11715.

2. Kawano, Y., et al., Stress-Strain Curves of Nafion Membranes in Acid and Salt Forms. Polímeros, 2002. 12: p. 96-101.

3. Molmeret, Y., et al., Towards Extrusion of Ionomers to Process Fuel Cell Membranes. Polymers, 2011. 3(3): p. 1126-1150.

4. Wu, J.F., et al., A review of PEM fuel cell durability: Degradation mechanisms and mitigation strategies. Journal of Power Sources, 2008. 184(1): p. 104-119.

5. Jin, Y.G., et al., Novel Nafion composite membranes with mesoporous silica nanospheres as inorganic fillers. Journal of Power Sources, 2008. 185(2): p. 664-669.

6. Lim, M.Y. and K. Kim, Sulfonated Poly(Arylene Ether Sulfone) and Perfluorosulfonic Acid Composite Membranes Containing Perfluoropolyether Grafted Graphene Oxide for Polymer Electrolyte Membrane Fuel Cell Applications. Polymers, 2018. 10(6).

7. Ketpang, K., K. Lee, and S. Shanmugam, Facile synthesis of porous metal oxide nanotubes and modified Nafion composite membranes for polymer electrolyte fuel cells operated under low relative humidity. ACS Applied Materials \& Interfaces, 2014. 6(19): p. 1673416744.

8. Jie, Z., T. Haolin, and P. Mu, Fabrication and characterization of self-assembled Nafion-SiO2-ePTFE composite membrane of PEM fuel cell. Journal of Membrane Science, 2008. 312(1-2): p. 41-47.

9. Ke, C.-C., et al., Investigation on sulfuric acid sulfonation of in-situ sol-gel derived Nafion/SiO2 composite membrane. international journal of hydrogen energy, 2011. 36(5): p. 3606-3613.

10. Hsu, W.Y. and T.D. Gierke, Ion-Transport and Clustering in Nafion Perfluorinated Membranes. Journal of Membrane Science, 1983. 13(3): p. 307-326.

11. Fujimura, M., T. Hashimoto, and H. Kawai, Small-Angle X-Ray-Scattering Study of Perfluorinated Ionomer Membranes .1. Origin of 2 Scattering Maxima. Macromolecules, 1981. 14(5): p. 1309-1315.

12. Rollet, A.-L., O. Diat, and G. Gebel, A new insight into Nafion structure. The Journal of Physical Chemistry B, 2002. 106(12): p. 3033-3036. 
13. Schmidt-Rohr, K. and Q. Chen, Parallel cylindrical water nanochannels in Nafion fuel-cell membranes. Nature materials, 2008. 7(1): p. 75-83.

14. Awatani, T., et al., Morphology of water transport channels and hydrophobic clusters in Nafion from high spatial resolution AFM-IR spectroscopy and imaging. Electrochemistry communications, 2013. 30: p. 5-8.

15. Xie, X., et al., Local probe and conduction distribution of proton exchange membranes. The Journal of Physical Chemistry B, 2007. 111(22): p. 6134-6140.

16. Dugger, J.W., et al., Ion movement in thin Nafion films under an applied electric field. Applied Physics Letters, 2018. 113(11): p. 113105.

17. Scott, W.W. and B. Bhushan, Use of phase imaging in atomic force microscopy for measurement of viscoelastic contrast in polymer nanocomposites and molecularly thick lubricant films. Ultramicroscopy, 2003. 97(1-4): p. 151-169.

18. Huang, Y.R., C.T. Chien, and C.L. Chen, A Molecular Dynamics Simulation Based Investigation of the Proton Conductivity of Anhydrous Pyrazole Doped Poly(Vinylphosphonic Acid) Composite System. Polymers, 2020. 12(12).

19. Tamayo, J. and R. Garcia, Deformation, contact time, and phase contrast in tapping mode scanning force microscopy. Langmuir, 1996. 12(18): p. 4430-4435.

20. Anczykowski, B., et al., Basic properties of dynamic force spectroscopy with the scanning force microscope in experiment and simulation. Ultramicroscopy, 1996. 66(3-4): p. 251-259.

21. Palermo, V., M. Palma, and P. Samorì, Electronic characterization of organic thin films by Kelvin probe force microscopy. Advanced materials, 2006. 18(2): p. 145-164.

22. Han, B., et al., Study on Micro Interfacial Charge Motion of Polyethylene Nanocomposite Based on Electrostatic Force Microscope. Polymers, 2019. 11(12): p. 2035.

23. Mélin, T., et al., Electric force microscopy of individually charged nanoparticles on conductors: an analytical model for quantitative charge imaging. Physical Review B, 2004. 69(3): p. 035321.

24. Deschler, J., J. Seiler, and J. Kindersberger, Detection of charges at the interphase of polymeric nanocomposites. IEEE Transactions on Dielectrics and Electrical Insulation, 2017. 24(2): p. 1027-1037.

25. Escorihuela, J., et al., Recent Progress in the Development of Composite Membranes Based on Polybenzimidazole for High Temperature Proton Exchange Membrane (PEM) Fuel Cell Applications. Polymers, 2020. 12(9).

26. Shen, Y., et al., Electrostatic force spectroscopy revealing the degree of reduction of individual graphene oxide sheets. Beilstein Journal of Nanotechnology, 2018. 9: p. 1146-1155.

27. Fumagalli, L., et al., Nanoscale capacitance imaging with attofarad resolution using ac current sensing atomic force microscopy. Nanotechnology, 2006. 17(18): p. 4581-4587.

28. Zhao, J.W. and K. Uosaki, Dielectric properties of organic monolayers directly bonded on silicon probed by current sensing atomic force microscope. Applied Physics Letters, 2003. 83(10): p. 2034-2036.

29. Kang, Y.H., et al., Conductance Mapping of Proton Exchange Membranes by Current Sensing Atomic Force Microscopy. Journal of Physical Chemistry B, 2009. 113(45): p. 15040-15046.

30. Flater, E.E., et al., Towards easy and reliable AFM tip shape determination using blind tip reconstruction. Ultramicroscopy, 2014. 146: p. 130-143.

31. Son, B., et al., Study of morphological characteristics on hydrophilicity-enhanced SiO2/Nafion composite membranes by using multimode atomic force microscopy. International Journal of Energy Research, 2019. 43(9): p. 4157-4169.

32. Kim, A.R., et al., Ameliorated Performance of Sulfonated Poly(Arylene Ether Sulfone) Block Copolymers with Increased Hydrophilic Oligomer Ratio in Proton-Exchange Membrane Fuel Cells Operating at 80\% Relative Humidity. Polymers, 2020. 12(9). 